\title{
Microarray analysis of hub genes and pathways in damaged cartilage tissues of knee
}

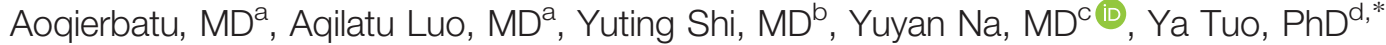

\begin{abstract}
The aim of this study was to identify genes and functional pathways associated with damaged cartilage tissues of knee using microarray analysis.

The gene expression profile GSE129147 including including 10 knee cartilage tissues from damaged side and 10 knee nonweightbearing healthy cartilage was downloaded and bioinformatics analysis was made.

A total of 182 differentially-expressed genes including 123 up-regulated and 59 down-regulated genes were identified from the GSE129147 dataset. Gene ontology and pathway enrichment analysis confirmed that extracellular matrix organization, collagen catabolic process, antigen processing and presentation of peptide or polysaccharide antigen, and endocytic vesicle membrane were strongly associated with cartilage injury. Furthermore, 10 hub differentially-expressed genes with a higher connectivity degree in protein-protein interactions network were found such as POSTN, FBN1, LOX, insulin-like growth factor binding proteins3, C3AR1, MMP2, ITGAM, CDKN2A, COL1A1, COL5A1.

These hub genes and pathways provide a new perspective for revealing the potential pathological mechanisms and therapy strategy of cartilage injury.

Abbreviations: BPs = biological processes, DEGs = differentially-expressed genes, FBN1 = fibrillin-1, GO = gene ontology, IGFBP = insulin-like growth factor binding protein, IGF-I = Insulin-like growth factor-I, LOX $=$ Iysyl oxidase, MCODE = molecular complex detection, $\mathrm{MHC}=$ major histocompatibility complex, $\mathrm{PPI}=$ protein-protein interactions.
\end{abstract}

Keywords: bioinformatics analysis, cartilage injury, differentially expressed genes, protein-protein interaction network

Editor: Mohammed Nader Shalaby.

$A$ and $A L$ contributed equally to this work.

This research was supported by the Natural Science Foundation of Inner Mongolia (Nos. 2020MS08119).

The authors have no conflicts of interest to disclose.

The datasets generated during and/or analyzed during the current study are publicly available.

a Department of Mongolian Osteopath, International Hospital of Mongolian Medicine, Saihan District, Hohhot, Inner Mongolia Autonomous Region, China,

${ }^{b}$ Cardiac Function Department, Cadre Health Care Center, Inner Mongolia Autonomous Region People's Hospital, Saihan District, Hohhot, Inner Mongolia Autonomous Region, China, ' Department of Arthroscopy and Sports Medicine, the Second Affiliated Hospital of Inner Mongolia Medical University, Huimin District, Hohhot, Inner Mongolia Autonomous Region, China, ${ }^{d}$ Department of Anesthesia, the Second Affiliated Hospital of Inner Mongolia Medical University, Huimin District, Hohhot, Inner Mongolia Autonomous Region, China.

* Correspondence: Ya Tuo, Department of anesthesia, the Second Affiliated Hospital of Inner Mongolia Medical University, Huimin District, Hohhot, Inner Mongolia Autonomous Region, China

(e-mail: 517720709@qq.com).

Copyright (๑) 2021 the Author(s). Published by Wolters Kluwer Health, Inc. This is an open access article distributed under the terms of the Creative Commons Attribution-Non Commercial License 4.0 (CCBY-NC), where it is permissible to download, share, remix, transform, and buildup the work provided it is properly cited. The work cannot be used commercially without permission from the journal.

How to cite this article: Aoqierbatu, Luo A, Shi Y, Na Y, Tuo Y. Microarray analysis of hub genes and pathways in damaged cartilage tissues of knee. Medicine 2021;100:37(e27183).

Received: 27 November 2020 / Received in final form: 20 August 2021 / Accepted: 21 August 2021

http://dx.doi.org/10.1097/MD.0000000000027183

\section{Introduction}

Cartilage is an avascular, noninnervated and alymphatic tissue with limited intrinsic repair potential. Articular cartilage lesions of the knee are commonly encountered in all ages. A retrospective study of 31,516 knee arthroscopies found $63 \%$ of their patients had cartilage injuries. ${ }^{[1]}$ Another retrospective study of 25,124 knee arthroscopies observed $60 \%$ of patients who suffered from knee pain had articular cartilage lesions. ${ }^{[2]}$ Due to its limited capacity to spontaneously repair largely, focal chondral defects can extend and progress into degeneration of the whole joint. ${ }^{[3,4]}$ So, focal chondral lesions of the knee are regarded as a potential risk factor for later osteoarthritis. ${ }^{[5]}$

Knee osteoarthritis is the result of imbalance between anabolic and catabolic metabolism of articular cartilage matrix; the main risk factor is aging, but the degeneration can be accelerated by mechanical factors, such as chondral lesions. ${ }^{[6]}$ Surgical treatments for chondral lesions including chondrocyte implantation, osteochondral transplantation, and microfracture, provide not fully satisfying outcomes. ${ }^{[6-9]}$ In this regard, understanding the molecular mechanisms and biological pathways that regulate chondrocyte metabolism and steady of cartilage matrix and gene therapy that enables the transfer of suitable genes into cartilage tissue is necessary for the treatment of cartilage lesions. Mori et $\mathrm{al}^{[10]}$ identified fibroblast growth factor-18 maintain the homeostasis of mature rats articular cartilage by decreasing glycosaminoglycan release and depletion from the cartilage, and enhancing proliferation of articular chondrocytes. Delivery of genes encoding for TGF- $\beta$, IGF-1, and BMP-2 by adenovirus vector to chondrocytes has been 
demonstrated to enhance proteoglycan synthesis by the cells in culture. ${ }^{[11,12]}$

Whole-genome microarray analysis is an efficient and highthroughput molecular technique for analyzing general genetic alterations in various tissues. ${ }^{[13-15]}$ For example, Aşık et al ${ }^{[16]}$ compared damaged and undamaged nonweight bearing cartilage and showed differentially-expressed genes (DEGs) and multiple biological processes (BPs) related to these DEGs. And, genome microarray combined with bioinformatic analysis can help us to better understand the underlying mechanisms that involved in development of disease.

The aim of this study was to identify DEGs and hub genes in damaged and healthy cartilage samples using bioinformatics methods and then to investigate the potential BPs and pathways of these changes.

\section{Materials and methods}

\subsection{Affymetrix microarray data}

The microarray data of GSE129147 was downloaded from National Center of Biotechnology Information Gene Expression Omnibus database (http://www.ncbi.nlm.nih.gov/geo/), based on GPL15207 platform ([PrimeView] Affymetrix Human Gene Expression Array; Affymetrix, Inc.), including 10 knee cartilage tissues from damaged side and 10 knee nonweight-bearing healthy cartilage. ${ }^{[16]}$

\subsection{Identification of DEGs in damaged and healthy cartilage samples}

The DEGs between damaged and healthy cartilage samples were screened by GEO2R (http://www.ncbi.nlm.nih.gov/geo/geo2r/). Genes with the adjusted $P$-value $<.05$ and $|\log \mathrm{FC}|>1$ or $|\log \mathrm{FC}|$ $<-1$ were regarded as the cutoff value for DEGs screening, which were the signature genes of damaged cartilage of knee.

\subsection{GO enrichment function and pathway analysis}

To determine the DEGs involved in BPs, cellular components, and molecular functions, gene ontology (GO) enrichment analysis were used with The Database for Annotation, Visualization and Integrated Discovery 6.8. ${ }^{[17]}$ Pathway analysis of DEGs was also performed with Database for Annotation, Visualization and Integrated Discovery online to learn the significantly altered metabolic pathways. ${ }^{[18]} \mathrm{GO}$ enrichment analysis $(P<.05, \mathrm{FDR}<0.05)$ and pathway analysis $(P<.05)$ of the DEGs was considered significant.

\subsection{Construction of the protein-protein interactions (PPI) network}

Search tool for the retrieval of interacting genes (STRING, version 11.0; http://string-db.org/, ELIXIR, Wellcome Genome Campus, Hinxton, Cambridgeshire, CB10 1SD, UK) was used to analyze the interaction between proteins for the DEGs with default parameters. ${ }^{[19]}$ Medium confidence $>0.4$ was defined as the cutoff criterion. Protein-protein interactions (PPI) network was visualized using Cytoscape (version 3.7.2, http://www. cytoscape.org/, the U.S. National Institute of General Medical Sciences) after downloading the raw image from search tool for the retrieval of interacting genes. ${ }^{[20]}$ In the network, the node represents a protein, the line represents the interaction, and the degree represents the number of interactions. And connectivity degree was analyzed by statistics in the network to obtain the important nodes, namely hub proteins. ${ }^{[21]}$

\subsection{Module analysis in the PPI network}

Molecular complex detection (MCODE) was used to discover dense clique-like structures within a network in the Cytoscape platform. Modules were extracted according to the criteria: degree cutoff $=2$, max. depth $=100, \mathrm{~K}$-Core $=2$, node score cutoff $=0.2$, and MCODE score $\geq 4$.

Table 1

The enriched GO terms and KEGG pathways for the down-regulated DEGs.

\begin{tabular}{|c|c|c|c|c|c|c|}
\hline Category & Term & Description & Count & Genes & $P$ value & FDR \\
\hline $\mathrm{BP}$ & G0:0030198 & Extracellular matrix organization & 10 & $\begin{array}{l}\text { B4GALT1, COL7A1, FBN1, POSTN, COL1A1, LOX, } \\
\text { COL8A1, LOXL1, ITGAM, COL5A1 }\end{array}$ & $1.38 \mathrm{E}-06$ & $2.00 \mathrm{E}-03$ \\
\hline $\mathrm{BP}$ & GO:0030574 & Collagen catabolic process & 7 & $\begin{array}{l}\text { COL7A1, MMP19, COL1A1, MMP14, COL8A1, MMP2, } \\
\text { COL5A1 }\end{array}$ & $1.42 \mathrm{E}-06$ & $2.00 \mathrm{E}-03$ \\
\hline $\mathrm{BP}$ & GO:0002504 & $\begin{array}{l}\text { Antigen processing and presentation of } \\
\text { peptide or polysaccharide antigen via } \\
\text { MHC class II }\end{array}$ & 5 & HLA-DRB1, HLA-DRB3, HLA-DRB4, HLA-DRB5, HLA-DRA & 1.99E-06 & $3.00 \mathrm{E}-03$ \\
\hline $\mathrm{CC}$ & GO:0030666 & Endocytic vesicle membrane & 7 & $\begin{array}{l}\text { WNT5B, HLA-DRB1, HLA-DRB3, HLA-DRB4, HLA-DRB5, } \\
\text { HLA-DRA, CD163 }\end{array}$ & $1.37 \mathrm{E}-06$ & $2.00 \mathrm{E}-03$ \\
\hline $\mathrm{CC}$ & GO:0042613 & MHC class II protein complex & 5 & HLA-DRB1, HLA-DRB3, HLA-DRB4, HLA-DRB5, HLA-DRA & $5.13 \mathrm{E}-06$ & $6.00 \mathrm{E}-03$ \\
\hline MF & GO:0042605 & Peptide antigen binding & 6 & $\begin{array}{l}\text { HLA-DRB1, HLA-DRB3, HLA-DRB4, HLA-DRB5, SLC7A5, } \\
\text { HLA-DRA }\end{array}$ & 4.27E-07 & $5.33 \mathrm{E}-04$ \\
\hline KEGG & hsa04145 & Phagosome & 9 & $\begin{array}{l}\text { ACTG1, ATP6V1A, HLA-DRB1, HLA-DRB3, HLA-DRB4, } \\
\text { HLA-DRB5, FCGR3A, ITGAM, HLA-DRA }\end{array}$ & $2.17 \mathrm{E}-05$ & $2.55 \mathrm{E}-02$ \\
\hline
\end{tabular}

$\mathrm{BPs}=$ biological processes, DEGs = differentially-expressed genes, $\mathrm{GO}=$ gene ontology, $\mathrm{MF}=$ molecular function, MHC = major histocompatibility complex 


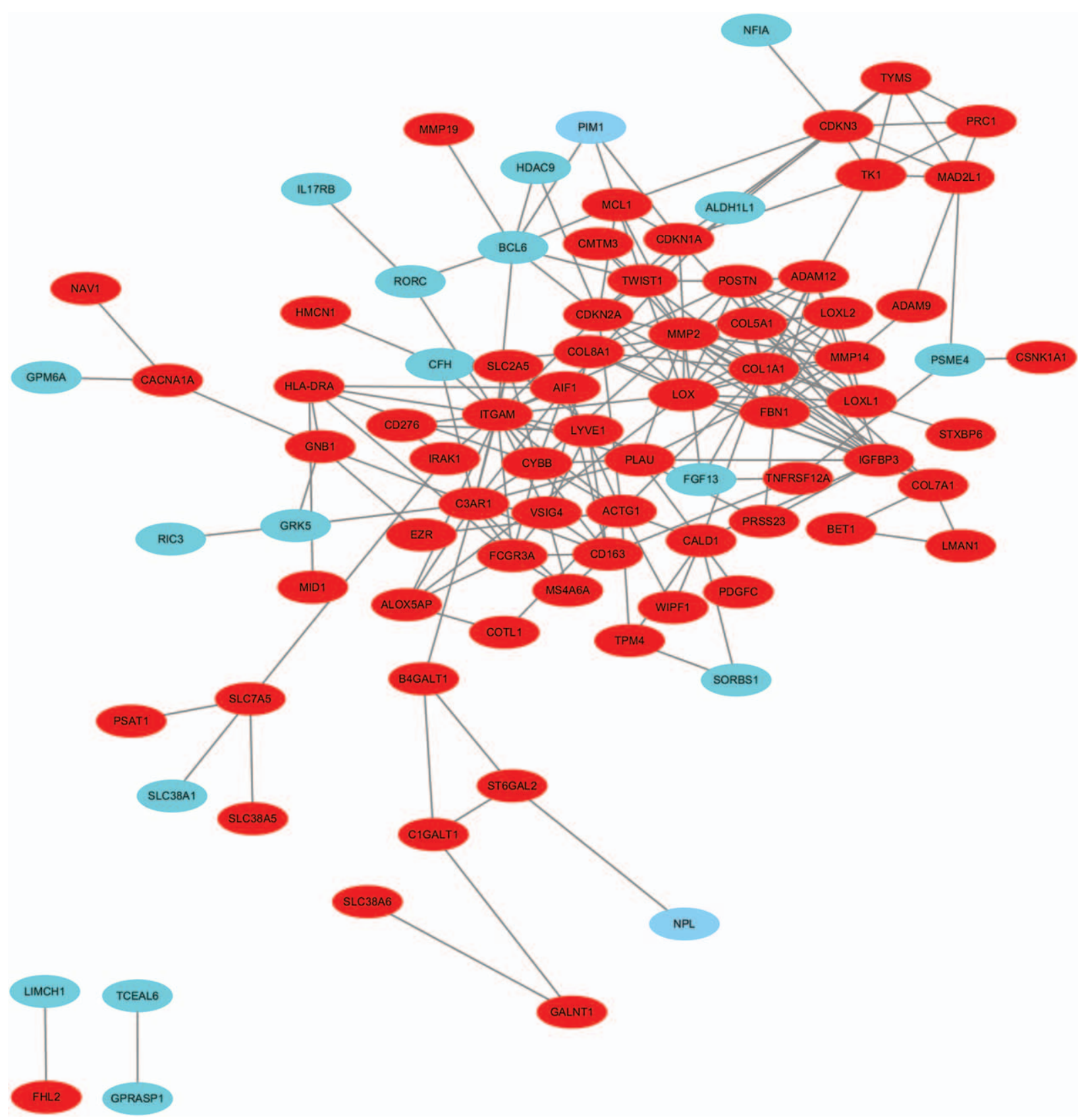

Figure 1. The constructed PPI network of DEGs. Node stand for the protein (gene), edge stand for the interaction of proteins. Red: up-regulated DEGs, Green: down-regulated DEGs. DEGs = differentially-expressed genes, PPI = protein-protein interactions.

\section{Results}

\subsection{DEGs identification and GO and pathway enrichment analysis}

In comparing damaged to healthy cartilage samples, a total of 182 DEGs including 123 up-regulated and 59 down-regulated genes were identified from the GSE129147 dataset. After GO functional enrichment analysis, 3 significant BP terms of upregulated DEGs including: extracellular matrix organization, collagen catabolic process, and antigen processing and presentation of peptide or polysaccharide antigen via major histocompatibility complex (MHC) class II; 3 significant CC terms of upregulated DEGs including: extracellular matrix, endocytic vesicle membrane, MHC class II protein complex; 1 significant molecular function terms of up-regulated DEGs is peptide antigen binding (Table 1). The up-regulated DEGs were enriched in 3 pathways such as Staphylococcus aureus infection, leishmaniasis, and phagosome. In terms of down-regulated DEGs, we did not find significant GO terms or KEGG pathways according to stated criteria.

\subsection{PPI network construction}

The PPI network was screened, comprising 132 nodes and 215 edges (Fig. 1). The top 10 DEGs with a higher connectivity degree are all up-regulated genes (Table 2), including POSTN, FBN1, LOX, insulin-like growth factor binding proteins (IGFBP) 3, C3AR1, MMP2, ITGAM, CDKN2A, COL1A1, COL5A1, 


\section{Table 2}

Results of connectivity degree of top 10 DEGs in the PPI network.

\begin{tabular}{lc}
\hline Genes & Degree \\
\hline MMP2 & 20 \\
ITGAM & 20 \\
COL1A1 & 17 \\
LOX & 14 \\
C3AR1 & 14 \\
POSTN & 12 \\
FBN1 & 11 \\
IGFBP3 & 11 \\
CDKN2A & 11 \\
COL5A1 & 11 \\
\hline
\end{tabular}

DEGS = differentially-expressed genes, FBN1 = fibrillin-1, IGFBP = insulin-like growth factor binding protein, $\mathrm{LOX}=$ lysyl oxidase, $\mathrm{PPI}=$ protein-protein interactions.

which are hub proteins. Among these genes, MMP2 (degree $=20)$ and ITGAM (degree $=20$ ) showed the highest node degree.

\subsection{Module analysis}

A total of 3 significant modules were selected with the parameter of MCODE score $\geq 4$. Module A (MCODE score $=8.889$ ), module $\mathrm{B}(\mathrm{MCODE}$ score $=5.000)$, module $\mathrm{C}(\mathrm{MCODE}$ score $=$ 4.000) were shown in Figure 2. Module A had 10 nodes involving 10 up-regulated gene (LOXL1, LOX, MMP14, LOXL2, MMP2, FBN1, COL5A1, POSTN, COL1A1, ADAM12); module B with
5 nodes and 10 edges involving 5 up-regulated gene (CDKN3, MAD2L1, PRC1, TYMS, TK1); module C with 4 nodes and 6 edges involving 4 up-regulated gene (FCGR3A, CD163, VSIG4, MS4A6A).

\section{Discussion}

In the present study, we explored the hub genes and pathways in damaged cartilage tissues of knee by bioinformatics methods. By comparing gene expression profiles between 10 knee cartilage from damaged side and 10 knee nonweight-bearing healthy cartilage tissues, we found 182 DEGs, including 123 up-regulated and 59 down-regulated genes in damaged cartilage tissues. Subsequently, GO enrichment analysis found that DEGs were associated with various BPs and molecular functions, such as extracellular matrix organization, collagen catabolic process, antigen processing and presentation of peptide or polysaccharide antigen via MHC class II, and endocytic vesicle membrane. In addition, a PPI network with these DEGs was constructed, and 10 hub genes, including POSTN, FBN1, LOX, IGFBP3, C3AR1, MMP2, ITGAM, CDKN2A, COL1A1, COL5A1, were identified as the hub genes with higher connectivity degree in damaged cartilage tissues.

In view of the results of GO terms enrichment analysis, we linked the up-regulated gene DEGs with extracellular matrix organization and collagen catabolic process. The structure and organization of cartilage's extracellular matrix are the primary determinants of normal function. Most diseases involving
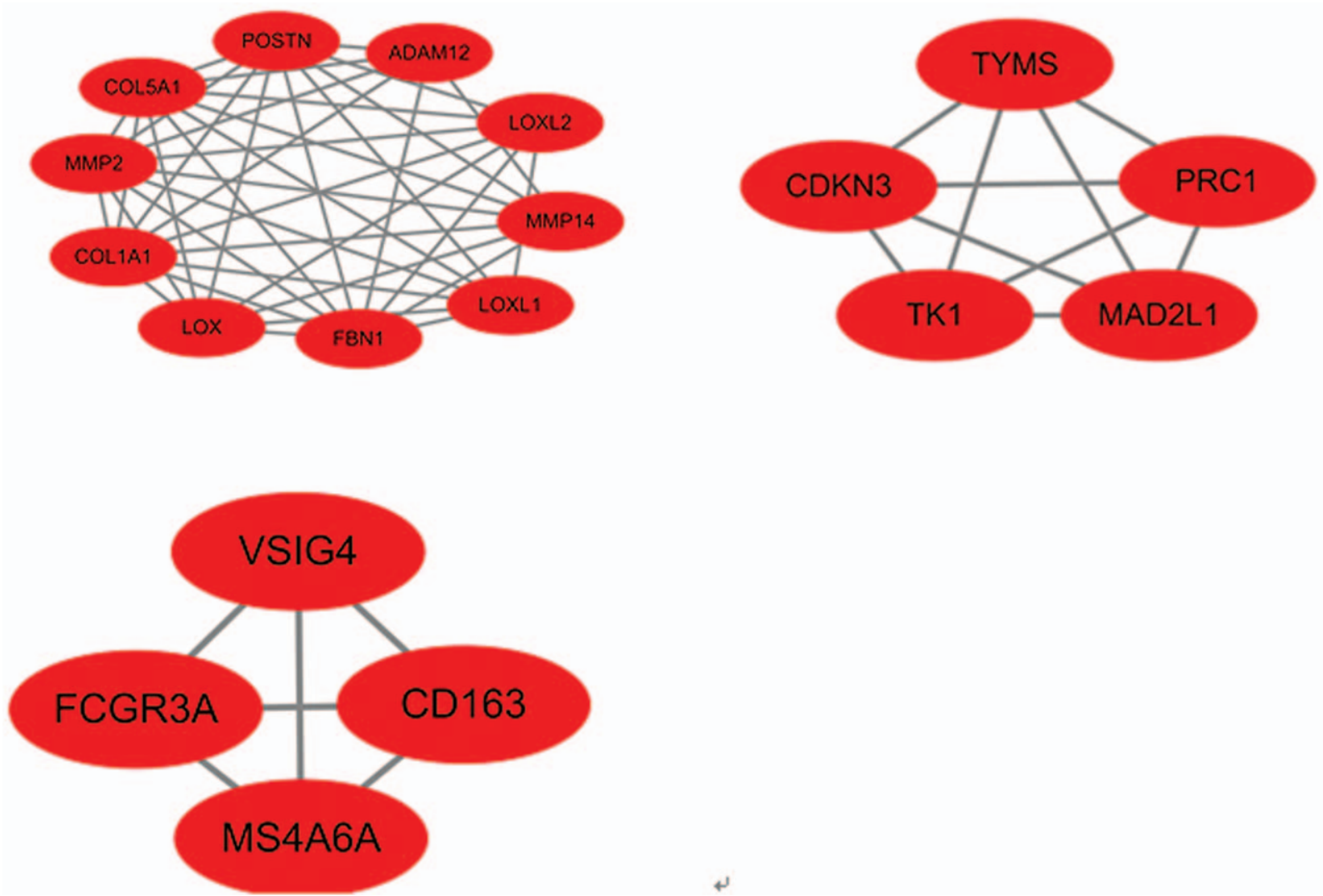

Figure 2. The 3 modules with MCODE score $>4$ selected from PPI network. The squares represent the DEGs in modules, and the lines show the interaction between the DEGs. This study is a secondary analysis. So, it does not involve research on humans. And the ethical approval is not necessary. DEGs $=$ differentiallyexpressed genes, FBN1 = fibrillin-1, LOX = lysyl oxidase, MCODE = molecular complex detection, PPI = protein-protein interactions. 
cartilage which lead to dramatic changes in the extracellular matrix. Well organized elastic networks have been found in the superficial zone of articular cartilage using immunohistochemistry or multiphoton microscopy together with histochemistry. Fibrillin-1 (FBN1) as the major component of elastic networks, mainly present in the uppermost superficial zone of articular cartilage. ${ }^{[22]}$ Collagens such as COL1A1 and COL5A1 and FBN1 were detectable in damaged cartilage tissues compared to undamaged controls in this study. The significant difference in expression levels of COL1A1, COL5A1, and FBN1 between damaged and undamaged sites of cartilage suggesting the attempt of chondrocytes for recovery of the damaged cartilage. Meanwhile, several matrix degrading proteases previously described to be linked to cartilage degeneration were upregulated in damaged cartilage, such as MMP2 and POSTN. Study considered POSTN as a catabolic protein that promotes cartilage degeneration through collagen and proteoglycan degradation in OA by up-regulating MMP-13 and ADAMTS4. ${ }^{[23]}$ These DEGs demonstrated the balance between synthetic and catabolic activities of chondrocytes is struggling after cartilage injuries.

Lysyl oxidase (LOX), is a copper-dependent amine oxidase, that catalyzes cross-linking of collagen and elastin, which plays an important role in biological functions of extracellular matrix. ${ }^{[24]}$ LOXL2 was visualized in tissues from human knee and hip joints by immunofluorescence. LOXL2 is upregulated in cartilage affected by $\mathrm{OA}$, may be a protective response that promotes anabolism while inhibiting specific catabolic responses in the pathophysiology of OA. ${ }^{[25]}$ LOXL3, a downstream of leptin, stimulates the apoptosis, but inhibits the autophagy of chondrocytes, is a potential therapy target for osteoarthritis. ${ }^{[26]}$ In this study, LOX is identified as an up-regulated gene in damaged cartilage tissues, which could promote cartilage maintenance.

IGFBP3 is 1 of 6 IGFBPs in humans. IGFBP3 modulates IGF signaling by binding to the 2 ligands (Insulin-like growth factor-I [IGF-I] and IGF-II) that primarily signal through IGF-I receptor. $^{[27]}$ In healthy articular cartilage from individuals without OA, IGF-I activates anabolism and inhibits catabolism of cartilage. Higher levels of IGFBP3 have been observed in OA cartilage, leading to the decrease of IGF-I's anabolic activity and could be partially responsible for the reduced responsiveness of OA cartilage to IGF-I. ${ }^{[28,29]}$ And a meta-analysis of genome-wide association studies of hip osteoarthritis found suggestive links between IGFBP3 and hip osteoarthritis and IGFBP3 overexpression induced cartilage catabolism and osteogenic differentiation. ${ }^{[30]}$ In this study, IGFBP3 is identified as an up-regulated gene in damaged cartilage tissues, which could promote cartilage degeneration.

Cells release into the extracellular environment different types of membrane vesicles called exosomes or microvesicles. Kim at al. found dendritic cells or vesicles derived from the dendritic cells expressing either secreted IL-4 or membrane-bound IL-4 are able to modulate the activity of APC and T cells in vivo through a MHC class II and partly Fas ligand/Fas-dependent mechanism, resulting in effective treatment of established collagen-induced arthritis. ${ }^{[31]}$ And Kim at al. also found exosomes derived from IL10-treated dendritic cells can suppress inflammation and collagen-induced arthritis. ${ }^{[32]}$ In this study, we linked the upregulated gene DEGs in antigen processing and presentation of peptide or polysaccharide antigen via MHC class II and endocytic vesicle membrane through GO terms enrichment analysis, which suggests extracellular vesicle and inflammation implicated in the pathogenesis of cartilage injuries.

In conclusion, our data provide a comprehensive bioinformatics analysis of DGEs and pathways, which may be involved in synthetic and catabolic metabolisms of chondrocytes after cartilage injuries. Nevertheless, additional experiments are needed to further validate the identified genes and pathways.

\section{Author contributions}

Data curation: Aoqierbatu None, Yuyan Na.

Formal analysis: Yuyan $\mathrm{Na}$, Ya Tuo.

Investigation: Aqilatu Luo, Yuting Shi.

Project administration: Aoqierbatu None, Yuyan $\mathrm{Na}$.

Resources: Ya Tuo.

Software: Yuting Shi.

Supervision: Ya Tuo.

Writing - original draft: Aoqierbatu None, Aqilatu Luo.

Writing - review \& editing: Yuyan $\mathrm{Na}$, Ya Tuo.

\section{References}

[1] Curl WW, Krome J, Gordon ES, Rushing J, Smith BP, Poehling GG. Cartilage injuries: a review of 31,516 knee arthroscopies. Arthroscopy 1997;13:456-60.

[2] Widuchowski W, Widuchowski J, Trzaska T. Articular cartilage defects: study of 25,124 knee arthroscopies. Knee 2007;14:177-82.

[3] Buckwalter JA, Mankin HJ, Grodzinsky AJ. Articular cartilage and osteoarthritis. Instr Course Lect 2005;54:465-80.

[4] Ossendorff R, Franke K, Erdle B, Uhl M, Südkamp NP, Salzmann GM. Clinical and radiographical ten years long-term outcome of microfracture vs. autologous chondrocyte implantation: a matched-pair analysis. Int Orthop 2019;43:553-9.

[5] Buckwalter JA, Mankin HJ. Articular cartilage: degeneration and osteoarthritis, repair, regeneration, and transplantation. Instr Course Lect 1998;47:487-504.

[6] Dall'Oca C, Cengarle M, Costanzo A, Giannini N, Vacchiano A, Magnan B. Current concepts in treatment of early knee osteoarthritis and osteochondral lesions; the role of biological augmentations. Acta Biomed 2017;88(4S):5-10.

[7] $\mathrm{Na}$ Y, Shi Y, Liu W, et al. Is implantation of autologous chondrocytes superior to microfracture for articular-cartilage defects of the knee? A systematic review of 5-year follow-up data. Int J Surg 2019;68:56-62.

[8] Tírico LEP, McCauley JC, Pulido PA, Bugbee WD. Lesion size does not predict outcomes in fresh osteochondral allograft transplantation. Am J Sports Med 2018;46:900-7.

[9] Frehner F, Benthien JP. Microfracture: state of the art in cartilage surgery? Cartilage 2018;9:339-45.

[10] Mori Y, Saito T, Chang SH, et al. Identification of fibroblast growth factor-18 as a molecule to protect adult articular cartilage by gene expression profiling. J Biol Chem 2014;289:10192-200.

[11] Smith P, Shuler FD, Georgescu HI, et al. Genetic enhancement of matrix synthesis by articular chondrocytes: comparison of different growth factor genes in the presence and absence of interleukin-1. Arthritis Rheum 2000;43:1156-64.

[12] Arai Y, Kubo T, Kobayashi K, et al. Adenovirus vector-mediated gene transduction to chondrocytes: in vitro evaluation of therapeutic efficacy of transforming growth factor-beta 1 and heat shock protein 70 gene transduction. J Rheumatol 1997;24:1787-95.

[13] Cui S, Zhang X, Hai S, et al. Molecular mechanisms of osteoarthritis using gene microarrays. Acta Histochem 2015;117:62-8.

[14] Li PCH. Overview of microarray technology. Methods Mol Biol 2016;1368:3-4.

[15] Zeng M, Liu J, Yang W, et al. Multiple-microarray analysis for identification of hub genes involved in tubulointerstial injury in diabetic nephropathy. J Cell Physiol 2019; doi: 10.1002/jcp.28313.

[16] Aşık MD, Gürsoy S, Akkaya M, Kozacı LD, Doğan M, Bozkurt M. Microarray analysis of cartilage: comparison between damaged and non-weight-bearing healthy cartilage. Connect Tissue Res 2020;61: 456-64. 
[17] Ashburner M, Ball CA, Blake JA, et al. Gene ontology: tool for the unification of biology. The Gene Ontology Consortium. Nat Genet 2000;25:25-9.

[18] Kanehisa M, Goto S. KEGG: Kyoto encyclopedia of genes and genomes. Nucleic Acids Res 2000;28:27-30.

[19] Szklarczyk D, Morris JH, Cook H, et al. The STRING database in 2017: quality-controlled protein-protein association networks, made broadly accessible. Nucleic Acids Res 2017;45:D362-8.

[20] Shannon P, Markiel A, Ozier O, et al. Cytoscape: a software environment for integrated models of biomolecular interaction networks. Genome Res 2003;13:2498-504.

[21] He X, Zhang J. Why do hubs tend to be essential in protein networks? PLoS Genet 2006;2:e88-188.

[22] Yu J, Urban JP. The elastic network of articular cartilage: an immunohistochemical study of elastin fibres and microfibrils. J Anat 2010;216:533-41.

[23] Attur M, Yang Q, Shimada K, et al. Elevated expression of periostin in human osteoarthritic cartilage and its potential role in matrix degradation via matrix metalloproteinase-13. FASEB J 2015;29: 4107-21.

[24] Smith-Mungo LI, Kagan HM. Lysyl oxidase: properties, regulation and multiple functions in biology. Matrix Biol 1998;16:387-98.

[25] Alshenibr W, Tashkandi MM, Alsaqer SF, et al. Anabolic role of lysyl oxidase like-2 in cartilage of knee and temporomandibular joints with osteoarthritis. Arthritis Res Ther 2017;19:179.
[26] Huang ZM, Du SH, Huang LG, Li JH, Xiao L, Tong P. Leptin promotes apoptosis and inhibits autophagy of chondrocytes through upregulating lysyl oxidase-like 3 during osteoarthritis pathogenesis. Osteoarthritis Cartilage 2016;24:1246-53.

[27] Jogie-Brahim S, Feldman D, Oh Y. Unraveling insulin-like growth factor binding protein-3 actions in human disease. Endocr Rev 2009;30: 417-37.

[28] Morales TI. The quantitative and functional relation between insulin-like growth factor-I (IGF) and IGF-binding proteins during human osteoarthritis. J Orthop Res 2008;26:465-74

[29] Doré S, Pelletier JP, DiBattista JA, Tardif G, Brazeau P, Martel-Pelletier J. Human osteoarthritic chondrocytes possess an increased number of insulin-like growth factor 1 binding sites but are unresponsive to its stimulation. Possible role of IGF-1-binding proteins. Arthritis Rheum 1994;37:253-63.

[30] Evans DS, Cailotto F, Parimi N, et al. Genome-wide association and functional studies identify a role for IGFBP3 in hip osteoarthritis. Ann Rheum Dis 2015;74:1861-7.

[31] Kim SH, Bianco NR, Shufesky WJ, Morelli AE, Robbins PD. Effective treatment of inflammatory disease models with exosomes derived from dendritic cells genetically modified to express IL-4. J Immunol 2007; 179:2242-9.

[32] Kim SH, Lechman ER, Bianco N, et al. Exosomes derived from IL-10treated dendritic cells can suppress inflammation and collagen-induced arthritis. J Immunol 2005;174:6440-8. 